\title{
Locally soluble groups with the restrictions on the generalized norms
}

\author{
T. Lukashova*
}

\author{
Communicated by I. Ya. Subbotin
}

\begin{abstract}
The author studies groups with given restrictions on norms of decomposable and Abelian non-cyclic subgroups. The properties of non-periodic locally soluble groups, in which such norms are nonidentity and have the identity intersection, are described.
\end{abstract}

\section{Introduction}

In group theory findings related to the study of the impact of properties of the different systems of the subgroups on the group are in focus. This direction includes findings when the restrictions are imposed on the different $\Sigma$-norms.

Let $\Sigma$ be the system of all subgroups of $G$ with a certain theoretical group property. The maximal subgroup of $G$ which normalizes every subgroup of $\Sigma$ is called $\Sigma$-norm of a group $G$. It is clear that the $\Sigma$-norm of a group $G$ coincides with the intersection of the normalizers of all subgroups included in $\Sigma$, and contains the center of a group.

In the case when $\Sigma$-norm of a group coincides with the group, all subgroups of $\Sigma$ are normal in the group (assuming that the system $\Sigma$ is non-empty). For the first time non-Abelian groups with such property

${ }^{*}$ The author expresses his deep gratitude to F. M. Lyman for his helpful suggestions during the preparation of this work.

2010 MSC: 20D25, 20E28.

Key words and phrases: locally soluble groups, non-periodic group, decomposable group, generalized norms of groups, norm of decomposable subgroups, norm of Abelian non-cyclic subgroups, non-Dedekind group. 
were considered in the second part of the XIX century by R. Dedekind, who characterized groups, all subgroups of which are normal (nowadays these groups are called Dedekind groups). However, the systematic study of groups with different systems of normal subgroups were continued only in the second part of the XX century, that slowed down the study of $\Sigma$-norms. So, the question on the study of the properties of groups, in which the $\Sigma$-norm is a proper subgroup, arises naturally.

For the first time such problem was formulated by R. Baer in 30s of the previous century for the system $\Sigma$ of all subgroups of a group [1]. Such $\Sigma$-norm was called the norm $N(G)$ of a group $G$ and denoted as the intersection of normalizers of all subgroups of a group $G$. Later the findings of R. Baer on the norm of a group were extended on the different systems of subgroups $\Sigma$ and on the different restrictions, which the $\Sigma$-norms satisfies (see e.g. [2]-[7]). It is clear that the norm $N(G)$ is contained in the other $\Sigma$-norms, which, in turn, can be regarded as its generalizations.

In this paper, we consider the relations between the norm of decomposable subgroups and the norm of Abelian non-cyclic subgroups of a group. The norm $N_{G}^{d}$ of decomposable subgroups of a group $G$ is the intersection of the normalizers of all decomposable subgroups of a group or group itself, if the system of such subgroups is empty [7]. Recall that a subgroup of a group $G$ is called decomposable if it can be representable in the form of the direct product of two non-trivial factors [8].

It is clear, that in the case when $N_{G}^{d}=G$, all decomposable subgroups are normal in a group $G$ or the system of such subgroups is empty. NonAbelian groups with such property were studied in [8] and called di-groups.

Obviously, the presence of decomposable subgroups in a group is directly related to the existence of decomposable Abelian subgroups, which in most cases are non-cyclic. So, the norm $N_{G}^{d}$ of decomposable subgroups of group $G$ is closely related to the norm $N_{G}^{A}$ of Abelian noncyclic subgroups.

The intersection of normalizers of all non-cyclic Abelian subgroups of a group $G$ (provided that the system of these subgroups is non-empty) is called the norm of non-cyclic Abelian subgroups of a group $G$ and denoted by $N_{G}^{A}$ (see e.g. [6,9]). If the norm $N_{G}^{A}$ contains at least one Abelian noncyclic subgroup, then each such a subgroup is normal in $N_{G}^{A}$. Non-Abelian groups with this property were studied by F. Lyman in [10] and called $\overline{H A}$-group. So, the norm of Abelian non-cyclic subgroups is Dedekind or non-Hamiltonian $\overline{H A}$-group.

The relations between these norms has been investigated in $[7,11,12]$ for quite broad classes of groups. In [7] it was proved that in locally finite 
groups, that contain an Abelian non-cyclic subgroup, one of the ratios holds: $N_{G}^{A} \subseteq N_{G}^{d}, N_{G}^{A} \supseteq N_{G}^{d}$.

In particular, it was found that a periodic locally nilpotent group has the non-Dedekind norm of decomposable subgroups if and only if it is a locally finite $p$-group and $N_{G}^{d}=N_{G}^{A}$. The same relations between norms can be traced in an arbitrarily locally finite group with the non-Dedekind locally nilpotent norm $N_{G}^{d}$ of decomposable subgroups (see [11]).

In [12] the study of the relations between the norm of decomposable subgroups and the norm of Abelian non-cyclic subgroups was continued in the class of non-periodic locally soluble groups. It was proved, if at least one of the norms $N_{G}^{A}$ or $N_{G}^{d}$ is non-Dedekind and the subgroup $N_{G}^{d}$ is infinite, then one of the following inclusions takes place: $N_{G}^{A} \subseteq N_{G}^{d}$ or $N_{G}^{d} \subseteq N_{G}^{A}$.

The purpose of the article is to study the properties of locally soluble groups in which the norm of decomposable subgroups and the norm of Abelian non-cyclic subgroups are nonidentity and have the identity intersection $N_{G}^{d} \cap N_{G}^{A}=E$.

\section{Preliminary results}

The next statements are actively used in the further research.

Lemma 1. ([7]) If a group $G$ contains a nonidentity $N_{G}^{d}$-admissible subgroup $H$ such that $N_{G}^{d} \cap H=E$, where $N_{G}^{d}$ is the norm of decomposable subgroups of $G$, then $N_{G}^{d}$ is Dedekind.

Lemma 2. ([6]) If a group $G$ contains an Abelian non-cyclic subgroup $H$ such that $N_{G}^{A} \cap H=E$, where $N_{G}^{A}$ is the norm of Abelian non-cyclic subgroups of $G$, then the norm $N_{G}^{A}$ is Dedekind (Abelian, if a group is non-periodic).

Lemma 3. ([6]) Let $G$ be a non-periodic group, $N_{G}^{A}$ be the norm of Abelian non-cyclic subgroups and a group $G$ contain a nonidentity $N_{G}^{A}$-admissible subgroup $H$ such that $N_{G}^{A} \cap H=E$. If the norm $N_{G}^{A}$ is non-periodic, then all infinite cyclic subgroups are normal in it.

The following statement reduces the study of groups, in which the norms $N_{G}^{A}$ of Abelian non-cyclic subgroups and the norm $N_{G}^{d}$ of decomposable subgroups are nonidentity and $N_{G}^{d} \cap N_{G}^{A}=E$, to the study of non-periodic groups. 
Theorem 1. If a locally soluble group $G$ contains an Abelian non-cyclic subgroup, the norm $N_{G}^{A}$ of Abelian non-cyclic subgroups and the norm $N_{G}^{d}$ of decomposable subgroups are nonidentity and $N_{G}^{d} \cap N_{G}^{A}=E$, then $G$ is a non-periodic group.

Proof. Suppose that $G$ is a periodic group. Then it is locally finite and either $N_{G}^{A} \supseteq N_{G}^{d}$ or $N_{G}^{A} \subseteq N_{G}^{d}$ by Theorem 1.4 [7], which contradicts the condition of the Theorem. Thus, $G$ is a non-periodic locally soluble group and the Theorem is proved.

Further we will consider only non-periodic groups in which the norms $N_{G}^{d}$ and $N_{G}^{A}$ are nonidentity and their intersection is identity. The existence of non-periodic groups with given restrictions on the norm of Abelian non-cyclic and the norm of decomposable subgroups is confirmed by the following examples.

Example 1. ([12], Example 3.5). Let $G=(\langle a\rangle \lambda B) \lambda\langle c\rangle$, where $|a|=p$ ( $p$ is prime, $p \neq 2$ ), $B$ be a group, isomorphic to an additive group of $p$-adic fractions, $B=B_{1}\langle x\rangle, x^{2} \in B_{1}, x^{-1} a x=a^{-1},\left[B_{1},\langle a\rangle\right]=E,|c|=$ $2,[c, a]=1, c^{-1} b c=b^{-1}$ for any element $b \in B$.

In this group the norm of decomposable subgroups $N_{G}^{d}=\langle a\rangle$ is a cyclic subgroup of prime order. At the same time, the norm of non-cyclic Abelian subgroups is non-Dedekind, $N_{G}^{A}=B_{1} \lambda\langle c\rangle$ and $N_{G}^{d} \cap N_{G}^{A}=E$.

\section{The main results}

The aim of this section is to study the properties of non-periodic locally soluble groups and the structure of the norms $N_{G}^{A}$ and $N_{G}^{d}$, provided that $N_{G}^{d} \cap N_{G}^{A}=E$. The first of the following theorems characterizes the groups with the non-Dedekind norm $N_{G}^{A}$, respectively, the second theorem describes groups in which the norm $N_{G}^{A}$ is the Dedekind.

Theorem 2. If a non-periodic locally soluble group $G$ has an Abelian non-cyclic subgroup, the norm $N_{G}^{A}$ of Abelian non-cyclic subgroups is nonDedekind, the norm $N_{G}^{d}$ of decomposable subgroups is nonidentity and $N_{G}^{d} \cap N_{G}^{A}=E$, then the following conditions take place:

1) $Z(G)=N(G)=E$, where $N(G)$ is the norm of $G$;

2) the norm of decomposable subgroups $N_{G}^{d}=\langle c\rangle$ is a cyclic group of a prime odd order $p$;

3) the norm $N_{G}^{A}$ of Abelian non-cyclic subgroups is a group of the type $N_{G}^{A}=A \lambda\langle b\rangle$, where $A$ is a group isomorphic to an additive group 
of $p$-adic fractions ( $p$ is prime, $(p, 2)=1),|b|=2$ and $b^{-1} a b=a^{-1}$ for any element $a \in A$;

4) any infinite cyclic subgroup has a nonidentity intersection with the norm $N_{G}^{A}$;

5) a group $G$ does not contain free Abelian subgroups of rank 2;

6) a group $G$ does not contain finite non-cyclic Abelian subgroups;

7) a group $G$ does not contain periodic non-cyclic locally cyclic subgroups;

8) the factor-group $G / N_{G}^{A}$ is periodic.

Proof. Let group $G$ satisfy the conditions of the theorem. Then the first statement of the theorem follows from the inclusions:

$$
Z(G) \subseteq N(G) \subseteq N_{G}^{d} \cap N_{G}^{A}=E .
$$

Let's show that the norm $N_{G}^{d}$ of decomposable subgroups is a cyclic group. Indeed, since $N_{G}^{d} \cap N_{G}^{A}=E$, the subgroup $N_{G}^{d}$ is Dedekind by Lemma 1. If $N_{G}^{d}$ contains non-cyclic Abelian subgroups, then the norm $N_{G}^{A}$ of Abelian non-cyclic subgroups is also Dedekind by Lemma 2, which contradicts the condition. Thus, $N_{G}^{d}$ does not contain non-cyclic Abelian subgroups.

Since $N_{G}^{d}$ is a Dedekind group by the proved above, $N_{G}^{d}$ is a finite group and its Sylow $p$-subgroups are cyclic for $p \neq 2$ and the Sylow 2-subgroup is either a cyclic group or the quaternion group. Taking into account the condition $Z(G)=E$, we make a conclusion that the order of the norm $N_{G}^{d}$ is not divided to 2 , because otherwise $N_{G}^{d}$ contains a central involution. Therefore, $N_{G}^{d}$ is a cyclic group, $N_{G}^{d}=\langle c\rangle$ and $(|c|, 2)=1$.

Considering that $\left|N_{G}^{d}\right|<\infty$, we obtain $\left[G: C_{G}\left(N_{G}^{d}\right)\right]<\infty$ and $x^{m} \in$ $C_{G}\left(N_{G}^{d}\right), m \in \mathbb{N}$ for an arbitrary element $x \in G,|x|=\infty$. Then the subgroup $\left\langle x^{m}, c\right\rangle$ is Abelian non-cyclic and $N_{G}^{A}$-admissible. If $\langle x\rangle \cap N_{G}^{A}=E$, then $\left\langle x^{m}, c\right\rangle \cap N_{G}^{A}=E$, and the norm $N_{G}^{A}$ is Dedekind by Lemma 2, which is impossible. Therefore, $N_{G}^{A}$ is a non-periodic group, and any infinite cyclic subgroup $\langle x\rangle$ of a group $G$ has a nonidentity intersection with the norm $N_{G}^{A}$.

Since the subgroup $\langle c\rangle$ is $N_{G}^{A}$-admissible and $\langle c\rangle \cap N_{G}^{A}=E$, all infinite cyclic subgroups are normal in $N_{G}^{A}$ by Lemma 3 . By the description of such groups (see [5]) $N_{G}^{A}$ is a group of the type $N_{G}^{A}=A\langle b\rangle$, where $A$ is a non-periodic Abelian group, $|b| \in\{2,4\}, b^{2} \in A$ and $b^{-1} a b=a^{-1}$ for any element $a \in A$.

Let's prove that the norm $N_{G}^{d}=\langle c\rangle$ of decomposable subgroups is a group of prime odd order $p$. Suppose that $\langle c\rangle \supseteq\left\langle c_{1}\right\rangle \times\left\langle c_{2}\right\rangle$, where 
$\left|c_{1}\right|=p,\left|c_{2}\right|=q,(p, q)=1, p$ and $q$ are odd prime. Then by the condition

$$
\left[N_{G}^{d}, N_{G}^{A}\right] \subseteq N_{G}^{A} \cap N_{G}^{d}=E,
$$

it follows that $\left|a c_{1}\right|=\infty$ for an arbitrary element $a \in A,|a|=\infty$. Since the subgroup $\left\langle a c_{1}, c_{2}\right\rangle$ is Abelian non-cyclic, it is $N_{G}^{A}$-admissible. Hence, the subgroup $\left\langle\left(a c_{1}\right)^{q}\right\rangle$ is also $N_{G}^{A}$-admissible. It is clear that the element $b \in N_{G}^{A}$ cannot be permutable with the element $\left(a c_{1}\right)^{q}$, because in this case $\left[\left(a c_{1}\right)^{q}, b\right]=\left[a^{q}, b\right]=1$, which is impossible. So,

$$
b^{-1}\left(a c_{1}\right)^{q} b=\left(a c_{1}\right)^{-q}=a^{-q} c_{1}^{q}=a^{-q} c_{1}^{-q}
$$

and $c_{1}^{2 q}=1$. We have a contradiction. Therefore, $|c|=p^{k}$, where $p$ is prime, $k \in \mathbb{N}$.

If $k>1$, then the subgroups $\langle a c\rangle \times\left\langle c^{p^{k-1}}\right\rangle$ and $\left\langle(a c)^{p}\right\rangle=\left\langle a^{p} c^{p}\right\rangle$ are $N_{G}^{A}$-admissible. Therefore, considering that $|a c|=\infty$ and $b^{-1} a b=a^{-1}$, where $b \in N_{G}^{A}$, we have

$$
b^{-1} a^{p} c^{p} b=\left(a^{p} c^{p}\right)^{-1}=a^{-p} c^{-p}=a^{-p} c^{p} .
$$

Then $c^{-p}=c^{p}$ and $c^{2 p}=1$, which is impossible. Therefore, $N_{G}^{d}=\langle c\rangle$, where $|c|=p, p \neq 2$.

Let's specify the structure of the subgroup $A \subseteq N_{G}^{A}$. Assume that $A$ is a mixed group and $T(A)$ is its periodic part. Since $N_{G}^{d} \cap N_{G}^{A}=E$, the group $G$ contains an indecomposable non-cyclic Abelian subgroup $H$ which is not $N_{G}^{d}$-admissible. Clearly, $H$ cannot be a complete group, because otherwise $H \subseteq C_{G}\left(N_{G}^{d}\right)$, which contradicts its choice. Therefore, $H$ is the incomplete non-cyclic Abelian torsion-free group of rank 1 .

Since $[\langle a\rangle, H] \subseteq T(A) \cap H=E$ for an arbitrary non-identity element $a \in T(A)$, the subgroup $\langle a\rangle \times H$ is Abelian decomposable and, therefore, $N_{G}^{d}$-admissible. Then by the condition

$$
\left[N_{G}^{d}, H\right] \subseteq N_{G}^{d} \cap(\langle a\rangle \times H)=E,
$$

the subgroup $H$ is also $N_{G}^{d}$-admissible, which is impossible. Thus, $A$ is an Abelian torsion-free group. Since $b^{2} \in A,|b|=2$ and $N_{G}^{A}=A \lambda\langle b\rangle$, where $b^{-1} a b=a^{-1}$ for an arbitrary element $a \in A$.

Suppose that the group $A$ contains a free abelian subgroup $\left\langle a_{1}\right\rangle \times\left\langle a_{2}\right\rangle$, where $\left|a_{1}\right|=\left|a_{2}\right|=\infty$. Since $H$ is a non-periodic Abelian torsion-free group of rank 1 and $N_{G}^{A} \cap H \neq E$ by the proved, at least one of the subgroups $\left\langle a_{1}\right\rangle$ or $\left\langle a_{2}\right\rangle$ has an identity intersection with $H$. Let $\left\langle a_{1}\right\rangle \cap H=$ $E$. Considering that $H$ is a $N_{G}^{A}$-admissible subgroup, let $a_{1}^{-1} h_{1} a_{1}=h_{2}$, 
where $h_{1}, h_{2} \in H$. Then by the condition $A \cap H \neq E$ we have $h_{1}^{k} \in A$ for some positive integer $k$. Thus, $a_{1}^{-1} h_{1}^{k} a_{1}=h_{1}^{k}=h_{2}^{k} h_{1}=h_{2}$ and $\left[\left\langle a_{1}\right\rangle, H\right]=E$.

It is clear that the subgroup $\left\langle a_{1}^{m}\right\rangle \times H$ is $N_{G}^{d}$-admissible for an arbitrary positive integer number $m$. Therefore, $H=\bigcap_{m=1}^{\infty}\left(\left\langle a_{1}^{m}\right\rangle \times H\right)$ is also $N_{G^{-}}^{d}$ admissible subgroup, which contradicts the choice of $H$. So, the subgroup $A$ does not contain free Abelian subgroups of rank 2 and is an Abelian torsion-free group of rank 1.

Let us consider the group

$$
G_{1}=N_{G}^{A} \times N_{G}^{d}=A \lambda\langle b\rangle \times\langle c\rangle,
$$

where $A$ is a torsion-free group of rank $1,|b|=2, b^{-1} a b=a^{-1}$ for any element $a \in A$ and $|c|=p, p \neq 2$. Since $N_{G}^{A}$ is a subgroup of the norm $N_{G_{1}}^{A}$ of Abelian non-cyclic subgroups of the group $G_{1}$ and $c \in Z\left(G_{1}\right)$, we have $G_{1}=N_{G_{1}}^{A}$ and $G_{1}$ is a $\overline{H A}$-group. By the description of such groups (see, e.g. [10]) $A$ is an infinite cyclic group or a group isomorphic to an additive group of $p$-adic fractions.

Assume that $A=\langle a\rangle$ is an infinite cyclic group. Then $\left(N_{G}^{A}\right)^{\prime}=\left\langle a^{2}\right\rangle \triangleleft$ $G, C=C_{G}\left(\left\langle a^{2}\right\rangle\right) \triangleleft G$ and $[G: C] \leqslant 2$. Since $b \notin C$, we have $G=C \lambda\langle b\rangle$, where $|b|=2$. By the results of [13] the centralizer $C$ contains all elements of infinite order of a group $G$ and all its Abelian non-cyclic subgroups. Moreover, the periodic part $T(C)$ of the subgroup $C$ is normal in $G$ and $C / T(C)$ is an Abelian torsion-free group of rank 1. Therefore, in this case the commutant $C^{\prime}$ is periodic and $C^{\prime} \subseteq T(C)$.

As $\left[N_{G}^{d}, N_{G}^{A}\right]=E$, it follows that $N_{G}^{d}=\langle c\rangle \subseteq T(C)$. Taking into account the non-Dedekindness of the norm $N_{G}^{A}$ and Lemma 2, we conclude that $T(C)$ does not contain Abelian non-cyclic subgroups, so $|T(C)|<\infty$. Let $u$ be a non-identity element of $T(C)$. Since the group $\left\langle u, a^{2}\right\rangle=$ $\langle u\rangle \times\left\langle a^{2}\right\rangle$ and its characteristic subgroup $\langle u\rangle$ are $N_{G}^{A}$-admissible,

$$
\left[\langle u\rangle, N_{G}^{A}\right] \subseteq N_{G}^{A} \cap\langle u\rangle=E .
$$

Hence, $\left[T(C), N_{G}^{A}\right]=E$ and $[u, b]=1$ for an arbitrary element $u \in T(C)$.

Suppose that $T(C)$ contains an involution $z$. Then subgroup $\langle b\rangle \times\langle z\rangle$ is Abelian non-cyclic and, therefore, $N_{G}^{A}$-admissible. Thus,

$$
\langle b\rangle=N_{G}^{A} \cap(\langle b\rangle \times\langle z\rangle) \triangleleft N_{G}^{A},
$$

which is impossible. So, $2 \notin \pi(T(C)$.

Let us prove that $T(C)=N_{G}^{d}$. Suppose for a contradiction that there exists an element $u \in T(C) \backslash\langle c\rangle$. Since $T(C)$ does not contain non-cyclic 
Abelian subgroups, then $|u| \neq p$ and the element $c$ is contained in each cyclic $p$-subgroup of the composite order. Therefore, if $|u|=p^{k}>p, k \in \mathbb{N}$, then $\langle c\rangle \subseteq\langle u\rangle$ and $[u, c]=1$. Now let $(|u|, p)=1$. Thus,

$$
[u, c] \in\left(\langle c\rangle \cap\left(\langle u\rangle \times\left\langle a^{2}\right\rangle\right)\right)=E
$$

and again $[u, c]=1$. Since the subgroup $\left\langle u a^{2}\right\rangle \times\langle c\rangle$ is Abelian non-cyclic and therefore $N_{G}^{A}$-admissible, we conclude that the subgroup $\left\langle u a^{2}\right\rangle^{p}=$ $\left\langle u^{p} a^{2 p}\right\rangle$ is also $N_{G}^{A}$-admissible. Then by the condition $[b, a] \neq 1$ we have that $\left[b, u^{p} a^{2 p}\right] \neq 1$ and

$$
b^{-1} u^{p} a^{2 p} b=\left(u^{p} a^{2 p}\right)^{-1}=u^{-p} a^{-2 p} .
$$

On the other hand, $b^{-1} u^{p} a^{2 p} b=u^{p} a^{-2 p}$, because $[u, b]=1$. Therefore, $u^{-p}=u^{p}$ and $u^{2 p}=1$, which is impossible. So, $T(C)=N_{G}^{d}=\langle c\rangle$, where $|c|=p, p \neq 2$.

Let $C_{1}=C_{C}\left(N_{G}^{d}\right)$ be the centralizer of the subgroup $N_{G}^{d}=\langle c\rangle$ in $C$. Since $C^{\prime} \subseteq\langle c\rangle$, the group $C_{1}$ is Abelian with the complementary subgroup $\langle c\rangle$, e.g., $C_{1}=\langle c\rangle \times Y$, where $Y$ is an Abelian torsion-free group of rank 1 . By the proved above $C$ contains all elements of infinite order of a group. Therefore, $H \subseteq C$, where $H$ is a non-cyclic Abelian torsion-free subgroup that is not $N_{G}^{d}$-admissible, and $C_{1} \neq C$.

By the cyclicity of the factor group $C / C_{1}$ and the previous considerations, we obtaine that the subgroup $Y$ is non-cyclic. Suppose that it contains an infinite sequence of subgroups

$$
\left\langle y_{1}\right\rangle \subset\left\langle y_{2}\right\rangle \subset \ldots \subset\left\langle y_{n}\right\rangle \subset \ldots,
$$

where $y_{n}=y_{n+1}^{k_{n+1}},\left(k_{n+1}, p\right)=1$ for all $n \in \mathbb{N}$. Then the isolator $I$ (see [14]) of the subgroup $\left\langle c y_{1}\right\rangle$ is non-cyclic and hence, $I$ is a $N_{G}^{A}$-admissible subgroup. Therefore,

$$
b^{-1}\left(c y_{1}\right) b \in\left(I \cap\left(\langle c\rangle \times\left\langle c y_{1}\right\rangle\right)\right)=\left\langle c y_{1}\right\rangle .
$$

Since $\left\langle y_{1}\right\rangle \cap\langle a\rangle \neq E$ and $b^{-1} y_{1} b=y_{1}^{-1}$, then $b^{-1} c y_{1} b=\left(c y_{1}\right)^{-1}=$ $c^{-1} y_{1}^{-1}=c y_{1}^{-1}$ and $c^{2}=1$, which is impossible. So, $Y$ does not contain such chains and hence is a group isomorphic to an additive group of $p$-adic numbers.

Let's prove that the subgroup $\langle c\rangle$ is complemented in $C$. By the proved above we have $1 \neq\left[C: C_{1}\right]=k$, where $k \mid(p-1)$. Since we can uniquely find the root of $k$ degree for each element of the subgroup $\langle c\rangle$ and $\langle c\rangle$ is complemented in its centralizer, it is also complemented in $C$ (Theorem 
1, [15]), e.g. $C=\langle c\rangle \lambda D$, where $D$ is an incomplete Abelian group of rank 1 .

It is obvious, that the group $G=(\langle c\rangle \lambda D) \lambda\langle b\rangle$ does not contain periodic Abelian non-cyclic subgroups, all mixed Abelian subgroups belong to the group $\langle c\rangle \lambda D$, contain $\langle c\rangle$ and are normal in $G$. Moreover, all tortionfree Abelian non-cyclic subgroups are contained either in the subgroup $D$ or in subgroups $g^{-1} D g, g \in G$, conjugated to this subgroup. Then the normalizer of each Abelian non-cyclic subgroup of rank 1 contains a subgroup $Y \triangleleft G$ and, as a consequence, the norm $N_{G}^{A}$ contains this subgroup, which contradicts the assumption of its structure. Therefore, $A$ cannot be an infinite cyclic group. So, $N_{G}^{A}=A \lambda\langle b\rangle$, where $A$ is a group isomorphic to an additive group of $p$-adic fractions, $(p, 2)=1,|b|=2$ and $b^{-1} a b=a^{-1}$ for any element $a \in A$.

By the proved above, every infinite cyclic subgroup has a nonidentity intersection with the norm $N_{G}^{A}$. On the other hand, the norm $N_{G}^{A}$ does not contain free Abelian subgroups of rank 2. So, the group $G$ also does not contain such subgroups. A similar statement holds for non-cyclic Abelian subgroups of finite order. Indeed, if a group $G$ contains finite Abelian non-cyclic subgroups, then their intersection with $N_{G}^{A}$, is a finite subgroup normal in $N_{G}^{A}$, which is impossible, or is an identity subgroup, which contradicts Lemma 2.

Suppose that $G$ contains a periodic non-cyclic locally cyclic subgroup $P$. If $P$ contains an infinite subgroup which has the identity intersection with the norm $N_{G}^{A}$, then the norm $N_{G}^{A}$ is Dedekind by Lemma 2, which is impossible. So, $P$ is a quasicyclic subgroup and $P \cap N_{G}^{A} \neq E$. But in this case $\left(P \cap N_{G}^{A}\right) \triangleleft N_{G}^{A}$, which contradicts the structure of the norm $N_{G}^{A}$. Therefore, the group $G$ does not contain periodic non-cyclic locally cyclic subgroups.

Finally, since the intersection $\langle x\rangle \cap N_{G}^{A}$ is nonidentity for an arbitrary element $x \in G,|x|=\infty$, the factor-group $G / N_{G}^{A}$ is periodic. The Theorem is proved.

Theorem 3. If a non-periodic locally soluble group $G$ has an Abelian noncyclic subgroup, the norm $N_{G}^{A}$ of Abelian non-cyclic subgroups is Dedekind, $N_{G}^{A} \neq E, N_{G}^{d} \neq E$ and $N_{G}^{d} \cap N_{G}^{A}=E$, then:

1) $Z(G)=N(G)=E$;

2) the norm $N_{G}^{A}$ of Abelian non-cyclic subgroups is an Abelian torsionfree group of rank 1 ;

3) the norm $N_{G}^{d}$ of decomposable subgroups is a cyclic group, $N_{G}^{d}=$ $\langle c\rangle,(|c|, 2)=1$. 
Proof. The first statement is proved in the same way as in Theorem 2. By the condition $N_{G}^{d} \cap N_{G}^{A}=E$ and Lemma 1 we have that the norm $N_{G}^{d}$ is Dedekind. Moreover, the group $G$ contains a non-primary, not $N_{G}^{A}$ admissible cyclic subgroup $\langle g\rangle$ and an indecomposable Abelian non-cyclic subgroup $H$, which is not $N_{G}^{d}$-admissible.

Suppose that the norm $N_{G}^{d}$ is non-periodic and an element $c \in N_{G}^{d}$ such that $|c|=\infty$ exists. Since the subgroup $\langle g\rangle$ is $N_{G}^{d}$-admissible, the subgroup $\left\langle g, c^{k}\right\rangle=\langle g\rangle \times\left\langle c^{k}\right\rangle$ is Abelian non-cyclic for some positive integer $k$, and therefore $N_{G}^{A}$-admissible. So, the subgroup $\langle g\rangle$ is also $N_{G}^{A}$-admissible, which contradicts its choice. Therefore, the norm $N_{G}^{d}$ is a periodic Dedekind group.

Assume that $N_{G}^{d}$ does not satisfy the minimal condition for Abelian subgroups. Then the intersection $C_{G}(g) \cap N_{G}^{d}$ contains non-cyclic Abelian subgroups $A_{1}$ and $A_{2}$ such that $\left(A_{1} \cup A_{2}\right) \cap\langle g\rangle=E$. Since the subgroups $A_{1} \times\langle g\rangle$ and $A_{2} \times\langle g\rangle$ are non-cyclic Abelian, they are $N_{G}^{A}$-admissible. So, the group

$$
\langle g\rangle=\left(A_{1} \times\langle g\rangle\right) \cap\left(A_{2} \times\langle g\rangle\right)
$$

is also $N_{G}^{A}$-admissible, which is impossible. This contradiction shows that $N_{G}^{d}$ is a group with the minimal condition for Abelian subgroups. Moreover, since the subgroup $N_{G}^{d}$ is Dedekind it follows from Corollary 4.2 [16] that $N_{G}^{d}$ is a finite extension of the direct product of a finite number of quasicyclic subgroups.

Let denote the subgroup generated by elements of the prime order of the norm $N_{G}^{d}$ by $\omega\left(N_{G}^{d}\right)$. By the proved above $\left|\omega\left(N_{G}^{d}\right)\right|<\infty$, so $[G$ : $\left.C_{G}\left(\omega\left(N_{G}^{d}\right)\right)\right]<\infty$. If an indecomposable non-cyclic Abelian subgroup $H$, which is not $N_{G}^{d}$-admissible, is complete, then $H \subseteq C_{G}\left(\omega\left(N_{G}^{d}\right)\right)$ and the group $B=H \cdot \omega\left(N_{G}^{d}\right)$ is Abelian. If $B$ is decomposable, then it is $N_{G}^{d}$-admissible. But in this case the subgroup

$$
B^{\left|\omega\left(N_{G}^{d}\right)\right|}=H^{\left|\omega\left(N_{G}^{d}\right)\right|}=H
$$

is also $N_{G}^{d}$-admissible, which contradicts its choice. Thus, $B$ is a nondecomposable Abelian group and as a consequence, $H$ is a quasicyclic $p$-group. So, $\omega\left(N_{G}^{d}\right) \subseteq H$ and $\left|\omega\left(N_{G}^{d}\right)\right|=p$. Since $Z(G)=E$ and the norm $N_{G}^{d}$ is Dedekind and contains an only one subgroup of prime order by the proved above, it is either a cyclic or a quasicyclic $p$-group. In both cases we conclude that $H \subseteq C_{G}\left(N_{G}^{d}\right)$. Therefore, the subgroup $H$ is $N_{G}^{d}$-admissible, which is impossible. Hence, $H$ is an incomplete non-cyclic Abelian torsion-free group of rank 1. 
Let's prove that the norm $N_{G}^{A}$ of Abelian non-cyclic subgroups is a torsion-free Abelian group. Indeed, otherwise, there exists a nonidentity element $x \in N_{G}^{A},|x|<\infty$. Then, taking into account that the norm $N_{G}^{A}$ is Dedekind and the subgroup $H$ is $N_{G}^{A}$-admissible, we have

$$
[\langle x\rangle, H] \subseteq T\left(N_{G}^{A}\right) \cap H=E,
$$

where $T\left(N_{G}^{A}\right)$ is the periodic part of the subgroup $N_{G}^{A}$. Therefore, the subgroup $\langle x\rangle \times H$ is decomposable Abelian and, as a consequence, $N_{G}^{d}$ admissible. But in this case

$$
\left[N_{G}^{d}, H\right] \subseteq N_{G}^{d} \cap(\langle x\rangle \times H)=E .
$$

Hence, $H$ is $N_{G}^{d}$-admissible subgroup, which contradicts its choice. So, $N_{G}^{A}$ is an Abelian torsion-free group.

If $N_{G}^{A} \cap H=E$, then $\left[N_{G}^{A}, H\right]=E$ and for any element $a \in N_{G}^{A},|a|=\infty$ the subgroup $\langle a\rangle \times H$ is Abelian decomposable and, hence, $N_{G}^{d}$-admissible. But then $\left[N_{G}^{d}, H\right] \subseteq N_{G}^{d} \cap(\langle a\rangle \times H)=E$, which is impossible, because in this case $H$ will be $N_{G}^{d}$-admissible subgroup. Therefore, $N_{G}^{A} \cap H \neq E$, and for an arbitrary element $h \in H$ there exists a non-zero integer $k$ such that $h^{k} \in N_{G}^{A}$.

Suppose that the norm $N_{G}^{A}$ contains a free Abelian subgroup $\left\langle a_{1}\right\rangle \times\left\langle a_{2}\right\rangle$, where $\left|a_{1}\right|=\left|a_{2}\right|=\infty$. Then by the proved at least one of the subgroups $\left\langle a_{1}\right\rangle$ or $\left\langle a_{2}\right\rangle$ has the identity intersection with $H$. Let $\left\langle a_{1}\right\rangle \cap H=E$. Since $H$ is a $N_{G}^{A}$-admissible subgroup, then $a_{1}^{-1} h_{1} a_{1}=h_{2}$, where $h_{1}, h_{2} \in H$. Moreover, by the condition $N_{G}^{A} \cap H \neq E$ we have $h_{1}^{k} \in N_{G}^{A}$ for some integer $k \neq 0$. Hence, $a_{1}^{-1} h_{1}^{k} a_{1}=h_{1}^{k}=h_{2}^{k}$, and $h_{1}=h_{2}$. Therefore, $\left[\left\langle a_{1}\right\rangle, H\right]=E$ and the subgroup $\left\langle a_{1}^{m}\right\rangle \times H$ is $N_{G}^{d}$-admissible for an arbitrary natural $m$. Thus, the subgroup $H=\bigcap_{m=1}^{\infty}\left(\left\langle a_{1}^{m}\right\rangle \times H\right)$ is also $N_{G^{-a d m i s s i b l e, ~ w h i c h ~}}^{d}$ contradicts its choice. So, the norm $N_{G}^{A}$ does not contain free abelian subgroups of rank 2 and is an Abelian torsion-free group of rank 1.

Let $\langle g\rangle$ be a non-primary subgroup, which is not $N_{G}^{A}$-admissible. It is clear that at least one of its Sylow $p$-subgroups is also not $N_{G}^{A}$-admissible. Let it be a subgroup $\langle g\rangle_{p}$, where $p$ is prime. Since the factor-group $G / C_{G}\left(N_{G}^{A}\right)$ is isomorphic to a subgroup of automorphisms of an Abelian torsion-free group of rank 1 with the periodic part of order 2 ([17], p. 294], we conclude that $\langle g\rangle_{p}=\langle g\rangle_{2}=\langle\bar{g}\rangle$ is a 2-group, $|\bar{g}|=2^{n}, n \in \mathbb{N}$.

Let's prove that all Sylow $p$-subgroups of $N_{G}^{d}$ are cyclic. Suppose that $N_{G}^{d}$ contains an elementary Abelian subgroup $N$ of order $p^{2}, p \neq 2$. Since

$$
[N,\langle\bar{g}\rangle] \subseteq\left(N_{G}^{d}\right)_{p} \cap\langle\bar{g}\rangle=E,
$$


where $\left(N_{G}^{d}\right)_{p}$ is a Sylow $p$-subgroup of the norm $N_{G}^{d}$, the subgroup $N \times\langle\bar{g}\rangle$ is an Abelian non-cyclic and $\langle\bar{g}\rangle$ is $N_{G}^{A}$-admissible as its characteristic subgroup, which is impossible. Therefore, any Sylow $p$-subgroup of the norm $N_{G}^{d}$ for $p \neq 2$ contains a unique subgroup of prime order, so, it is a cyclic or a quasicyclic $p$-group.

Suppose that the norm $N_{G}^{d}$ contains quasicyclic $p$-subgroup $P$ for some prime $p \neq 2$. Then $P \times\langle\bar{g}\rangle$ is Abelian non-cyclic, and hence, $N_{G}^{A}$-admissible group. Thus, the subgroup $\langle\bar{g}\rangle$ is $N_{G}^{A}$-admissible, which contradicts its choice. So, any Sylow $p$-subgroup of the norm $N_{G}^{d}$ is cyclic for $p \neq 2$.

Let us consider the Sylow 2-subgroup $\left(N_{G}^{d}\right)_{2}$ of the norm $N_{G}^{d}$. If $\left(N_{G}^{d}\right)_{2} \cap\langle\bar{g}\rangle=E$, then for an arbitrary element $c \in\left(N_{G}^{d}\right)_{2}$ the subgroup $\langle c, \bar{g}\rangle=\langle c\rangle \times\langle\bar{g}\rangle$ is Abelian non-cyclic, and therefore, is $N_{G}^{A}$-admissible. Then $\left[\langle\bar{g}\rangle, N_{G}^{A}\right] \subseteq(\langle c\rangle \times\langle\bar{g}\rangle) \cap N_{G}^{A}=E$, which is impossible. Thus, $\left(N_{G}^{d}\right)_{2} \cap\langle\bar{g}\rangle \neq E$.

Let's denote the lower layer of the Sylow 2-subgroup $\left(N_{G}^{d}\right)_{2}$ by $M$ and consider the group $G_{2}=\langle\bar{g}\rangle M$. Let $M$ be a non-cyclic group. Then by the condition $\langle\bar{g}\rangle \triangleleft G_{2}$ we have $[\langle\bar{g}\rangle, M] \subseteq M \cap\langle\bar{g}\rangle=\left\langle c_{1}\right\rangle$, where $c_{1} \in M,\left|c_{1}\right|=2$. As $\langle\bar{g}\rangle=C_{G_{2}}(\langle\bar{g}\rangle)$, it follows $M=\left\langle c_{1}\right\rangle \times\left\langle c_{2}\right\rangle$ and $\left[\langle\bar{g}\rangle,\left\langle c_{2}\right\rangle\right]=\left\langle c_{1}\right\rangle$. Then by $M \triangleleft G,\left[G: C_{G}(M)\right]=2$ and $\bar{g} \notin C_{G}(M)$, we conclude that $G=C_{G}(M)\langle\bar{g}\rangle$. However, in this case $c_{1} \in Z(G)$, which is impossible. Therefore, the lower layer of $\left(N_{G}^{d}\right)_{2}$ contains one involution, which again contradicts the condition $Z(G)=E$. So, $2 \notin \pi\left(N_{G}^{d}\right)$ and $N_{G}^{d}=\langle c\rangle,(|c|, 2)=1$. The Theorem is proved.

The following example confirms the existence of groups satisfying the conditions of Theorem 3 and generalizes Example 3.4 of [12]. Let's note that the order of the norm of decomposable subgroups in this case can be a composite number (unlike the groups satisfying the conditions of Theorem 2).

Example 2. Let $G=(\langle a\rangle \lambda B) \lambda\langle c\rangle$, where $|a|=m>1,(m, 2)=1$, $B$ is a group isomorphic to an additive group of $q$-adic fractions, $q$ is prime, $(q, 2 m)=1, B=B_{1}\langle x\rangle, x^{2} \in B_{1}, x^{-1} a x=a^{-1},\left[B_{1},\langle a\rangle\right]=E$, $|c|=2,[c, a]=1$ and $c^{-1} b c=b^{-1}$ for any element $b \in B$.

In this group, all periodic decomposable subgroups have the order $2 d$, $d \mid m, d>1$ and are groups of the form $\left\langle a^{\frac{m}{d} s} c b_{1}^{k}\right\rangle$, where $b_{1} \in B_{1},(s, d)=$ $1, k \in\{0,1\}$. Thus, all nonperiodic decomposable subgroups are mixed and contained in the group $B_{1} \times\langle a\rangle$ and, hence, they are normal in $G$. Since $N_{G}\left(\left\langle a^{\frac{m}{d} s} c b_{1}^{k}\right\rangle\right)=\left\langle a c b_{1}^{k}\right\rangle$, we conclude that $N_{G}^{d}=\langle a\rangle$. 
Let's determine the norm $N_{G}^{A}$ of non-cyclic Abelian subgroups of the group $G$. It is obvious that $G$ does not contain periodic non-cyclic Abelian subgroups but all mixed Abelian subgroups contain $\left\langle a^{\frac{m}{d} s}\right\rangle$, and are subgroups of the group $B_{1} \times\langle a\rangle$. It is easy to prove that all these subgroups are normal in $G$. Further, all non-cyclic Abelian subgroups of rank 1 are contained either in the subgroup $B$ or in the subgroups $g^{-1} B g, g \in G$, conjugate to this subgroup, or in the group $B_{1} \times\langle a\rangle$. Let's consider an infinite sequence of subgroups in $B_{1}$ :

$$
\left\langle y_{1}\right\rangle \subset\left\langle y_{2}\right\rangle \subset \ldots \subset\left\langle y_{n}\right\rangle \subset \ldots
$$

where $y_{n}=y_{n+1}^{k_{n+1}},\left(k_{n+1}, m\right)=1$ for all $n \in \mathbb{N}$.

It is easy to prove that the isolator $I$ of the subgroup $\left\langle a y_{1}\right\rangle$ is non-cyclic because the root of the element $a$ of any power mutually prime with $m$ exists. Moreover, $N_{G}(I)=B_{1} \times\langle a\rangle$. Since $N_{G}(B)=B \lambda\langle c\rangle$, we conclude that $N_{G}^{A}=B_{1}$ is a torsion-free Abelian group of rank 1 and $N_{G}^{d} \cap N_{G}^{A}=E$.

\section{References}

[1] R. Baer, Der Kern, eine charakteristische Untergruppe, Comp. Math., N.1, 1934, pp. 254-283.

[2] F.M. Lyman, T.D. Lukashova, M.G. Drushlyak, Generalized norms of groups Algebra discrete math., V. 22, N. 1, 2016, pp.48-80.

[3] M. de Falco, F. de Giovanni, L. A. Kurdachenko, C. Musella, The Metanorm and its Influence on the Group Structure, J. Algebra, V. 506, 2018, pp. 76-91.

[4] M. de Falco, F. de Giovanni, L. A.Kurdachenko, C. Musella, The metanorm, a characteristic subgroup: embedding properties, J. Group Theory, V. 21, Is. 5, 2018, pp. 847-864.

[5] F.M. Lyman, T.D. Lukashova, On norm of infinite cyclic subgroups in nonperiodic groups, Bull. P. M. Masherov Vitebsk State Univ., N. 4, 2006, pp.108-111.

[6] T.D. Lukashova, M.G. Drushlyak, F.M. Lyman, Conditions of Dedekindness of generalized norms in non-periodic groups, Asian-European Journal of Mathematics, V. 12, Is.1, 2019, P. 1950093 (11 pages), doi.org/10.1142/S1793557119500931.

[7] F.N. Liman, Lukashova T. D. On the norm of decomposable subgroups in locally finite groups Ukr. Math. J., V. 67, N. 4, 2015, pp.542-551.

[8] F.N. Liman, Groups in which every decomposable subgroup is invariant, Ukr. Math J., V. 22, N. 6, 1970, pp.625-631.

[9] T.D. Lukashova, On norm of Abelian non-cyclic subgroups in infinite locally finite p-groups $(p \neq 2)$, Bull. Taras Shevchenko National Univ. Kiev, N. 3, 2004, pp.35-39.

[10] F.M. Lyman, Non-peridic groups with some systems of invariant subgroups, Algebra and Logic, V. 7, N. 4, 1968, pp.70-86. 
[11] T.D. Lukashova, Infinite locally finite groups with the locally nilpotent non-Dedekind norm of decomposable subgroups, Communications in Algebra, V. 48, N. 3, 2016, pp. 1052-1057, doi.org/10.1080/00927872.2019.1677683

[12] F.N. Liman, T.D. Lukashova, On the norm of decomposable subgroups in the non-periodic groups, Ukr. Math. J., V. 67, N. 12, 2016, pp.1900-1912.

[13] F. N. Lyman, M. G. Drushlyak, On non-periodic groups without free Abelian subgroups of rank 2 with non-Dedekind norm of Abelian non-cyclic subgroups, Bulletin of University of Dnipropetrovsk, N. 6, 2011, pp.83-97.

[14] A. G. Kurosh, Theory of Groups [in Russian], Nauka, Moscow, 1967.

[15] J.D. Dixon, Complements of normal subgroups in infinite groups, Pros. London.Math.Soc., V. 17, N. 3, 1967, pp.431-446.

[16] S. N. Chernikov, Groups with given properties of system of subgroups, Moscow, Nauka, 1980.

[17] L. Fuchs, Infinite Abelian Groups, V.2. [in Russian], Moscow, Mir, 1977.

\section{CONTACT INFORMATION}

Tetiana Lukashova Taras Shevchenko National University of Kyiv, Volodymyrska 60, Kyiv, Ukraine, 01033 E-Mail(s): tanya.lukashova2015@gmail.com

Received by the editors: 15.01.2020. 